\title{
How "free" are free neutrons in neutron-star crusts and what does it imply for pulsar glitches?
}

\author{
N. Chamel \\ Institute of Astronomy and Astrophysics, Université Libre de Bruxelles, CP 226, \\ Boulevard du Triomphe, B-1050 Brussels, Belgium
}

\begin{abstract}
The neutron superfluid permeating the inner crust of mature neutron stars is expected to play a key role in various astrophysical phenomena like pulsar glitches. Despite the absence of viscous drag, the neutron superfluid can still be coupled to the solid crust due to non-dissipative entrainment effects. Entrainment challenges the interpretation of pulsar glitches and suggests that a revision of the interpretation of other observed neutron-star phenomena might be necessary.
\end{abstract}

Keywords. stars: neutron, dense matter, equation of state, gravitation, hydrodynamics, stellar dynamics, (stars:) pulsars: general, stars: rotation

\section{Introduction}

Pulsars are among the most accurate clocks in the universe, the delays associated with their spin-down being at most of a few milliseconds per year. Nevertheless, some pulsars have been found to exhibit sudden increases in their rotational frequency $\Omega$. These "glitches", whose amplitude varies from $\Delta \Omega / \Omega \sim 10^{-9}$ up to $\sim 10^{-5}$ (see e.g. Section 12.4 in Chamel \& Haensel 2008). The long relaxation times following the first observed glitches and the glitches themselves hinted at the presence of superfluids in neutron-star interiors (Baym et al. 1969, Packard 1972). In fact, neutron-star superfluidity had been predicted by Migdal (1959) based on the microscopic theory of superconductivity developed by Bardeen, Cooper and Schrieffer two years earlier. Subsequently Ginzburg \& Kirzhnits (1964) estimated the critical temperature for neutron superfluidity and suggested that the interior of a neutron star could be threaded by an array of quantized vortices. Anderson \& Itoh (1975) advanced the seminal idea that pulsar glitches are triggered by the sudden unpinning of such vortices in the neutron-star crust. Their scenario found some support from laboratory experiments in superfluid helium (Campbell 1979, Tsakadze \& Tsakadze 1980). Further developments aimed at explaining the post-glitch relaxation by the motion of vortices (Pines \& Alpar 1985, Jones 1993). In the meantime, Alpar et al. (1984) argued that the core of a neutron star (supposed to contain superfluid neutrons and superconducting protons) is unlikely to play any role in glitch events. Large pulsar glitches are still usually interpreted as sudden transfers of angular momentum between the neutron superfluid in the crust and the rest of the star. The confidence in this interpretation comes from i) the regularity observed in many glitching pulsars and ii) the fact that the estimated ratio of the moment of inertia $I_{s}$ of the superfluid component driving glitches to the total stellar moment of inertia $I$ is about $I_{s} / I \sim 1-2 \%$ at most, as expected if only the crustal superfluid is involved (Link et al. 1999). 


\section{Crustal entrainment}

Even though the neutron superfluid in the crust can flow without friction, it can still be entrained by nuclei, as first shown by Carter et al. (2005). Indeed, unbound or "dripped" neutrons can be Bragg reflected by the crustal lattice in which case they cannot propagate and are therefore trapped in the crust. Unlike viscous drag, this entrainment effect is non-dissipative. Neutron diffraction is a well-known phenomenon, which has been routinely exploited to probe the structure of materials. Unlike the neutron beams used in terrestrial experiments, neutrons in neutron-star crusts are highly degenerate. Due to the Pauli exclusion principle, they must all have different (Bloch) wave vectors. As a result, they are simultaneously scattered in different directions. The strength of entrainment is therefore determined by the way all unbound neutrons are diffracted. This can be characterized by the density $n_{n}^{\mathrm{c}}$ of conduction neutrons, i.e. neutrons that are effectively "free" to move with a different velocity than that of nuclei. Equivalently, entrainment effects can be embedded in an effective neutron mass $m_{n}^{\star}=m_{n} n_{n}^{\mathrm{f}} / n_{n}^{\mathrm{c}}$ where $m_{n}$ is the bare neutron mass and $n_{n}^{\mathrm{f}}$ the density of unbound neutrons. Neutron conduction has been systematically studied in all regions of the inner crust using the band theory of solids (see Chamel 2012). The neutron superfluid has thus been found to be very strongly entrained by the crust, especially in the region with average baryon densities $\bar{n} \sim 0.02-0.03 \mathrm{fm}^{-3}$.

\section{Implications for pulsar glitches}

According to a popular interpretation, pulsar glitches are due to sudden transfers of angular momentum between the neutron superfluid permeating the crust and the rest of the star. Due to entrainment, the angular momentum $J_{\mathrm{s}}$ of the superfluid depends not only on the angular velocity $\Omega_{\mathrm{s}}$ of the superfluid, but also on the (observed) angular velocity $\Omega$ of the star and can be expressed as (see Chamel \& Carter 2006)

$$
J_{\mathrm{S}}=I_{\mathrm{ss}} \Omega_{\mathrm{s}}+\left(I_{\mathrm{s}}-I_{\mathrm{ss}}\right) \Omega,
$$

Chamel \& Carter (2006) showed that the product of the fractional moments of inertia $I_{\mathrm{S}} / I$ and $I_{\mathrm{s}} / I_{\mathrm{ss}}$ should obey the following constraint

$$
\frac{\left(I_{\mathrm{S}}\right)^{2}}{I I_{\mathrm{ss}}} \geqslant \mathcal{G}, \quad \mathcal{G} \equiv \frac{1}{t} \sum_{i} \frac{\Delta \Omega_{i}}{|\dot{\Omega}|},
$$

where the sum is over all glitches observed during the time $t$ and $\dot{\Omega}$ is the observed average pulsar spin-down rate. A statistical study of glitching pulsars leads to $\mathcal{G} \simeq 1.7 \%$ (see Lyne et al. 2000). If entrainment is neglected, $I_{\mathrm{ss}}=I_{\mathrm{s}}$ so that $J_{\mathrm{s}}=I_{\mathrm{s}} \Omega_{\mathrm{s}}$ and $\left(I_{\mathrm{S}}\right)^{2} /\left(I I_{\mathrm{SS}}\right)$ reduces to $I_{\mathrm{s}} / I$. Approximating this ratio by the fractional moment of inertia of the crust $I_{\text {crust }} / I$, the constraint (3.2) was found to be easily satisfied for any realistic equation of state yielding plausible values for the neutron-star mass $M$ and radius $R$ (see Link et al. 1999). On the other hand, taking entrainment into taken account (see Chamel), we have found that $\left(I_{\mathrm{S}}\right)^{2} /\left(I I_{\mathrm{ss}}\right) \simeq 0.17 I_{\text {crust }} / I$. Observations of large pulsar glitches and (3.2) require that the fractional moment of inertia of the crust exceed $\sim 10 \%$. The ratio $I_{\text {crust }} / I$ can be estimated using the approximate expression of Lattimer \& Prakash (2000). The resulting fractional moment of inertia of the crust is shown in Fig. 1 for different neutron-star masses and radii using the neutron-star crust model of Onsi et al. (2008).

This analysis implies that very active glitching pulsars should have an unusually small mass, significantly below the canonical value of $1.4 M_{\odot}\left(M_{\odot}\right.$ being the mass of our Sun). The existence of low mass neutron stars is not excluded, but such stars are not expected to be formed in a type II supernova explosion (see e.g. Strobel \& Weigel 2001). Therefore, 
pulsars exhibiting large glitches should not be found in supernova remnants. This prediction is contradicted by the emblematic Vela pulsar, whose association with a supernova remnant is well established.

\section{Conclusions}

Due to entrainment effects, the neutron superfluid in neutron-star crusts does not carry enough angular momentum to explain large pulsar glitches. On the other hand, Alpar et al. (1984) argued that the neutron superfluid in the core is also strongly coupled to the crust. The solution to this problem requires a closer examination of crustal entrainment and crust-core coupling. The presence of nuclear "pastas" at the crust bottom, quantum and thermal fluctuations of ions about their equilibrium positions, crystal defects, impurities and more generally any kind of disorder would presumably reduce the number of entrained neutrons. Further work is needed to confirm these expectations. However, Sotani et al. (2012) have recently argued that quasi-periodic oscillation observed in giant flares from soft gamma-ray repeaters restrict the existence of pastas (if any) to a very narrow crustal region. Moreover, observations of the initial cooling in persistent soft X-ray transients are consistent with a low level of impurities in the crust (see Shternin, Yakovlev \& Haensel 2007, Brown \& Cumming 2009) and this level is unlikely to be higher in non-accreting neutron stars like Vela. Incidentally these observations provided another proof for crustal superfluidity. On the other hand, the strong crust-core coupling assumed here could be much weaker, especially if protons form a type II superconductor (see Sedrakian et al. 1995). The observed rapid cooling of the neutron star in Cassiopeia A has recently provided strong evidence for core neutron superfluidity and proton superconductivity, but not on its type (see Page et al. 2011, Shternin 2011). Link (2003) showed that type II superconductivity is incompatible with observations of long-period precession in pulsars. In the meantime, the work of Lyne et al. (2010) has cast some doubt on the interpretation of long-period precession. In fact, proton superconductivity might be neither of type I nor of type II (see Babaev 2009). In addition, neutron-star

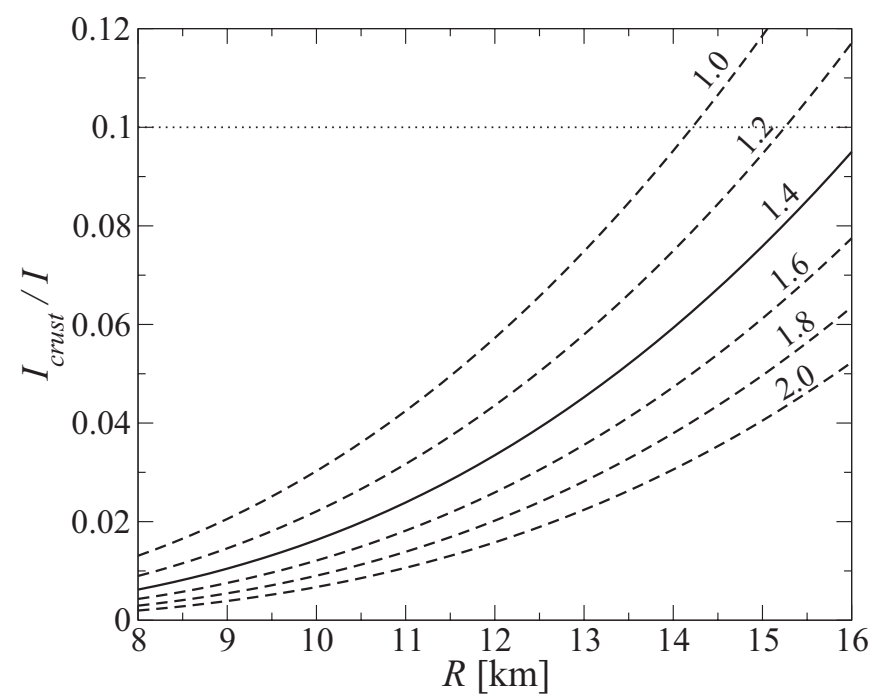

Figure 1. Fractional moment of inertia of neutron-star crusts for different neutron-star masses (in solar masses) and radii. The horizontal dotted line indicates the lowest value consistent with Vela pulsar glitches. 
cores might contain other particle species with various superfluid and superconducting phases (see e.g. Oertel \& Buballa 2006). This warrants further studies. But if glitches are induced by the core of a neutron star, it will be challenging to explain the observed regularity of glitches and the fact that $\mathcal{G} \lesssim 2 \%$.

\section{Acknowledgements}

This work was supported by FNRS (Belgium) and CompStar, a Research Networking Programme of the European Science Foundation. The author thanks B. Link and A. Alpar for valuable discussions.

\section{References}

Alpar, M. A., Langer, S. A., \& Sauls, J. A. 1984, Astrophys. J., 282, 533

Anderson, P. W. \& Itoh, N. 1975, Nature, 256, 25

Babaev, E. 2009, Phys. Rev. Lett., 103, 231101

Baym, G., Pethick, C. J., \& Pines, D. 1969, Nature, 224, 673

Brown, E. F. \& Cumming, A. 2009, Astrophys. J., 698, 1020

Campbell, L. J. 1979, Phys. Rev. Lett., 43, 1336

Carter, B., Chamel, N., \& Haensel, P. 2005, Nucl. Phys. A, 748, 675

Chamel, N. 2005, Nucl.Phys. A, 747, 109

Chamel, N. \& Carter, B. 2006, Mon.Not.Roy.Astron.Soc., 368, 796

Chamel, N. \& Haensel, P. 2008, "Physics of Neutron Star Crusts", Living Rev. Relativity 11, 10. http://www.livingreviews.org/lrr-2008-10

Chamel, N. 2012, Phys. Rev. C, 85, 035801

Chamel, N., submitted

Ginzburg, V. L. \& Kirzhnits, D. A. 1964, Zh. Eksp. Teor. Fiz. 47, 2006

Jones, P. B. 1993, Mon. Not. R. Astron. Soc., 263, 619

Lattimer, J. M. \& Prakash, M. 2000, Phys.Rep., 333, 121

Link, B., Epstein, R. I., \& Lattimer, J. M. 1999, Phys. Rev. Lett., 83, 3362

Link, B. 2003, Phys. Rev. Lett. 91, 101101

Lyne, A. G., Shemar, S. L., \& Smith, F. G. 2000, Mon. Not. R. Astron. Soc., 315, 534

Lyne, A. G., Hobbs, G., Kramer, M., Stairs, I., \& Stappers, B. 2010, Science, 329, 408

Migdal, A. B. 1959, Nucl. Phys., 13, 655

Oertel, M. \& Buballa, M. 2006, in: "Color superconducting quark matter and the interior of neutron stars" in Superdense QCD Matter and Compact Stars, ed. by D. Blaschke and D. Sedrakian. Proceedings of the NATO Advanced Research Workshop, 27 September - 4 October, 2003, in Yerevan, Armenia, Springer, Dordrecht, The Netherlands, p. 187

Onsi, M., Dutta, A. K., Chatri, H., Goriely, S., Chamel, N., \& Pearson, J. M. 2008, Phys. Rev. $C, 77,065805$

Packard, R. E. 1972, Phys. Rev. Lett., 28, 1080

Page, D., Prakash, M., Lattimer, J. M., \& Steiner, A. W. 2011, Phys. Rev. Lett., 106, 081101

Pines, D. \& Alpar, M. A. 1985, Nature, 316, 27

Ruderman, M. 1972, Annu. Rev. Astron. Astrophys., 10, 427

Sedrakian, A. D., Sedrakian, D. M., Cordes, J. M., \& Terzian, Y. 1995, Astrophys. J., 447, 324

Shternin, P. S., Yakovlev, D. G., Haensel, P., \& Potekhin, A. Y. 2007, Mon.Not.Roy.Astron.Soc., $382, \mathrm{~L} 43$

Shternin, P. S., Yakovlev, D. G., Heinke, C. O., Ho, W. C. G., \& Patnaude, D. J. 2011, Mon.Not.Roy.Astron.Soc., 412, L108

Sotani, H., Nakazato, K., Iida, K., \& Oyamatsu, K. 2012, Phys. Rev. Lett., 108, 201101

Tsakadze, J. S. \& Tsakadze, S. J. 1980, J. Low Temp. Phys., 39, 649

Strobel, K. \& Weigel, M. K. 2001, Astron. Astrophys., 367, 582 